\section{SAT0691 INFLUENCE OF SMOKING IN THE EXPRESSION OF CHRONIC PERIODONTITIS AND ANTI-CITRULLINATED PROTEINS ANTIBODIES IN RHEUMATOID ARTHRITIS}

J. González Febles ${ }^{1}$, J.L. Garnier Rodríguez ${ }^{2}$, F. Sánchez Alonso ${ }^{3}$, S. Dadlani ${ }^{2}$, Y. Barrios ${ }^{4}$, S. Bustabad ${ }^{5}$, F. Díaz González ${ }^{5}$, M. Sanz Alonso ${ }^{1}$, B. Rodríguez Lozano ${ }^{5} .{ }^{1}$ Periodontology, UCM, Madrid; ${ }^{2}$ Odontology/Periodontology, Dental Clinic Dr Garnier, Santa Cruz de Tenerife; ${ }^{3}$ Research Unit, Sociedad Española de Reumatologia, Madrid; ${ }^{4}$ Immunology Section. Central Laboratory;

${ }^{5}$ Rheumatology, Hospital Universitario de Canarias, Tenerife, Spain

Background: Environmental, genetic and epigenetic factors can induce citrullination of structural peptides by the enzyme PAD,which induce anti-citrullinated protein antibodies (ACPA) preceding RA. Among the environmental factors are cigarette smoke, infections, such as $P$. gingivalis in periodontitis (PD) and Prevotella copri of intestinal microflora, and silica dust. Given the implication of these two exogenous factors, tobacco and PD, in citrullination, and tobacco enhancer factor in PD, we studied:

Objectives: 1. The risk of smoking for developing advanced PD in patients with RA. 2. Possible influence of smoking on the expression of severe PD and ACPA in RA patients.

Methods: Observational, descriptive, cross-sectional study in RA patients older $\tan 18 y$.o.(ACR/EULAR 2010), with $\geq 4$ teeth, without tooth cleaning nor antibiotic intake 6 months previously. Socio-demographic and anthropometric variables included smoking status, social indicators such as Graffar scale, stress level, annual dental prophylaxis, and co-morbidities such as diabetes mellitus, dyslipidemia, ischemic cardiovascular disease. Serum ACPA detection: semiquantification Ab IgG against citrullinated peptides (ELISA) with Immunoscan CCPlus ${ }^{\circledR}$ test kit. Eurodiagnostica: positive $>25$; ACPA title stratification: Low (25-75), moderate $(76-300)$ and high ( $>300)$. Periodontal parameters: plaque index (PI), Bleeding on probing (Bop), probing pocket depth, recession, clinical attachment level (CAL). CAL loss was categorized according to European Workshop 2005 (Tonetti) ${ }^{1}$ : T level 0 (abscence), TL1 (mild), TL2 (severe). Statistical analysis: t-student, Kruskal Wallis, Chi-cuadrado by Stata program 13.1

Results: We studied 187 patients, F/M 78.6\%/21.4\%, mean age 54.4 y.o. Followup time 8.8 y.o. RF+ $74.2 \%$, ACPA positive in $114 / 168$ patients $(67.86 \%)$. Smoking habit:Current smoker $(19.25 \%)$, former smoker $(24.6 \%)$; low socioeconomical status $(36.4 \%)$ / relative poverty (33.7\%).PD was observed in $97.3 \%$ : TL1 $52.4 \%$, TL2 44.9\%. A "risk gradient" was observed for PD related to smoking habit: former smoker OR 1.62 (95\% Cl 0.81-3.27), $\mathrm{p}=0.174$; smokers, OR 2.27 (95\% Cl 1.05$4.91), p=0.037$. When analyzing the influence of smoking on PD development according to ACPA profile, a gradient effect of developing severe PD was observed from former smokers OR 2.37 (IC95\% 0.52-7.64) to current smokers OR 6.99 (IC95\% 1.53-32.07) $(\mathrm{p}=0.029)$ in ACPA(-) patients. This relationship was not observed in ACPA $(+)$ patients $(\mathrm{p}=0.383)$

Conclusions: 1. There is a "risk gradient" to develop PD in RA in relation to past or current exposure to tobacco, so that, although not significant, former smokers are at greater risk than non-smokers, and current smokers have a significant risk 2.3 times higher. 2. This risk gradient is shown in ACPA (-) patients, but not in ACPA (+) patients, which suggests an independent relationship between PD and ACPA (+) RA.

References:

[1] Tonetti MS, et al. J Clin Periodontol 2005.

Disclosure of Interest: None declared

DOI: 10.1136/annrheumdis-2017-eular.5201

\section{SAT0692 PREDICTORS AND PERSISTENCE OF UNACCEPTABLE PAIN DURING THE FIRST YEAR OF RHEUMATOID ARTHRITIS IN SWEDEN}

H. Westerlind ${ }^{1}$, M.E. Schelin ${ }^{1}$, R. Altawil ${ }^{2}$, L. Klareskog ${ }^{3}$, L. Alfredsson ${ }^{1}$, J. Lampa ${ }^{3} \cdot{ }^{1}$ Institute of Environmental Medicine; ${ }^{2}$ Rheumatology Unit, Department of Medicine, Center for Molecular Medicine (CMM): ${ }^{3}$ Rheumatology Unit, Department of Medicine, Center for Molecular Medicine (CMM), Karolinska Institutet, Stockholm, Sweden

Background: Pain is a dominant symptom in rheumatoid arthritis (RA). Objectives: Investigate unacceptable pain (VAS-pain $>40$ ) during the first year of the disease and whether it can be predicted from baseline disease characteristics. Methods: The cohort included all incident RA cases from the Swedish populationbased case-control Epidemiological Investigation of Rheumatoid Arthritis study (EIRA), who also were in the Swedish Rheumatology Register. Unacceptable pain was defined as scoring $40 \mathrm{~mm}$ or above on the pain visual analog scale (VAS) (i.e. not reaching the patient acceptable symptom state (PASS) (1)), and the proportion of patients going in and out of PASS was traced over the first year. Association between baseline parameters, divided into quartiles, and unacceptable pain at one year was assessed using modified Poisson regression and expressed as risk ratios with $95 \%$ confidence intervals $(95 \% \mathrm{Cl}$ ), adjusted for sex and age at diagnosis. Results: A total of 2808 patients were included in the study and $33.8 \%$ of the patients presented with PASS (i.e.VAS pain below 40) at inclusion. If a patient had PASS at any given visit, there was over $70 \%$ chance that the patient remained in PASS at the following visit. The most common PASS pattern (25.6\%), was to present with unacceptable pain, reach PASS at the 3 month visit, and then remain in PASS. However, one year after diagnosis, only two thirds of the patients had PASS. Higher disability (measured as HAQ) at baseline was significantly and independently associated with an increased risk for unacceptable pain at one year (for the highest quartile of $\mathrm{HAQ}$; RR=1.97 [95\% $\mathrm{Cl}: 1.60-2.42]$ ). Also high tender joint count at baseline was associated with an increased risk for unacceptable pain; $R R=1.40$ [95\% Cl: 1.18-1.65] for the highest quartile, whereas high swollen joint count at baseline was associated with a decreased risk; RR=0.79 [95\% Cl: $0.66-0.95]$ for the highest quartile.

Conclusions: The results highlight the need for efficient pain treatment strategies early in the disease.

References:

[1] Tubach F, Ravaud P, Martin-Mola E, Awada H, Bellamy N, Bombardier C, et al. Minimum clinically important improvement and patient acceptable symptom state in pain and function in rheumatoid arthritis, ankylosing spondylitis, chronic back pain, hand osteoarthritis, and hip and knee osteoarthritis: Results from a prospective multina. Arthritis Care Res (Hoboken) 2012;64:1699-1707.

Acknowledgements: We would like to acknowledge the EIRA study group and the EIRA data collectors.

Disclosure of Interest: None declared

DOI: 10.1136/annrheumdis-2017-eular.6133

\section{SAT0693 SERUM URIC ACID LEVELS AND HORMONE REPLACEMENT THERAPY TYPE: A RETROSPECTIVE CASE-CONTROL STUDY OF POSTMENOPAUSAL WOMEN}

J.H. Jung, J.-H. Kim, Y.H. Lee, G.G. Song, S.J. Choi. Internal Medicine, Korea University Medical Center, Seoul, Korea, Republic Of

Background: Serum uric acid levels increase in postmenopausal women but decrease when hormone replacement therapy (HRT) is administered. However, no study has evaluated the effects of different types of HRT on serum uric acid levels

Objectives: We examined whether estrogen monotherapy, estrogen-progestogen combination therapy, and tibolone use affected serum uric acid levels in this population.

Methods: We performed a retrospective case-control study of postmenopausal women. From 2005 to 2015, postmenopausal women measured serum uric acid levels more than twice were included. Patients were grouped according to HRT regimen: estrogen monotherapy, estrogen-progestogen combination therapy, or tibolone. The control group did not receive HRT. Differences in serum uric acid levels were examined in each group. Our analysis was adjusted to accommodate different follow-up intervals for individual patients. Multiple variables were adjusted using the Tukey-Kramer method. Age, body mass index, hypertension, diabetes mellitus, dyslipidemia, alcohol consumption, smoking status, and co-medications were also adjusted.

Results: In the control group, the serum uric acid level increased to $0.86 \pm 0.27$ $\mathrm{mg} / \mathrm{dL}$ (least squares mean \pm standard error). In comparison, after adjusting for multiple variables, the serum uric levels in the estrogen-progestogen combination

Table 1. Degrees of changes in serum uric acid levels by hormone replacement therapy type

\begin{tabular}{|c|c|c|c|c|c|c|c|}
\hline & \multicolumn{2}{|c|}{ Estrogen mono } & \multicolumn{2}{|c|}{ Estrogen-progestogen } & \multicolumn{2}{|c|}{ Tibolone } & \multirow{2}{*}{$\begin{array}{c}\text { Control } \\
\Delta \text { uric acid }\end{array}$} \\
\hline & $\Delta$ uric acid & $P$ & $\Delta$ uric acid & $P$ & $\Delta$ uric acid & $\mathrm{P}$ & \\
\hline & $0.64 \pm 0.26$ & 1.000 & $-0.50 \pm 0.17$ & $<0.001$ & $0.16 \pm 0.18$ & 0.097 & $0.67 \pm 0.13$ \\
\hline & & & & & & & \\
\hline Model II & & 0.9 & & & & 0.0 & \\
\hline & & & & & & & \\
\hline & & 0.986 & & & & 0.195 & $0.74 \pm 0.26$ \\
\hline Model V & $0.85 \pm 0.35$ & 1.000 & $-0.41 \pm 0.29$ & $<0.001$ & $0.26 \pm 0.27$ & 0.096 & $0.86 \pm 0.27$ \\
\hline
\end{tabular}

All models that included crude values were adjusted for follow-up intervals. Model I: adjusted age. Model II: adjusted age and BMI. Model III: adjusted age, BMI, HTN, DM, and dyslipidemia. Model IV: adjusted age, BMI, HTN, DM, dyslipidemia, alcohol consumption, and smoking status. Model V: adjusted age, BMI, HTN, DM, dyslipidemia, alcohol consumption, smoking status and co-medications.

1.5

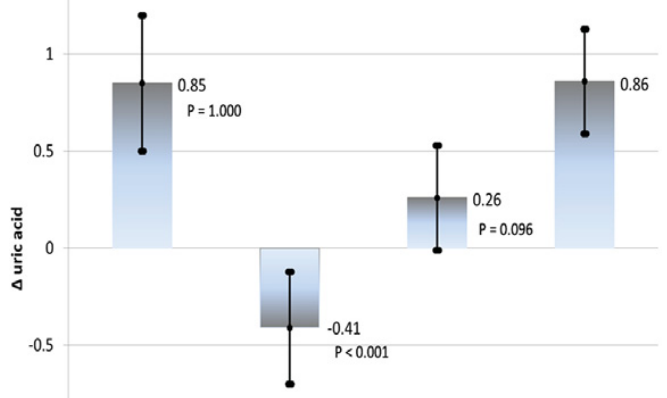

$-1.5$

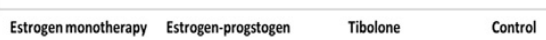

